\title{
Reaction cycling for efficient kinetic analysis in flow
}

\author{
Ryan J. Sullivan and Stephen G. Newman*[a]
}

\begin{abstract}
A reactor capable of efficiently collecting kinetic data in flow is presented. Conversion over time data is obtained by passing a discrete reaction slug back-and-forth between two residence coils, with analysis performed each time the solution is passed from one coil to the other. In contrast to a traditional steady state continuous flow system, which requires upwards of $5 \times$ the total reaction time to obtain reaction progress data, this design achieves much higher efficiency by collecting all data during a single reaction. In combination with minimal material consumption (reactions performed in $300 \mu \mathrm{L}$ slugs), this represents an improvement in efficiency for typical kinetic experimentation in batch as well. Application to kinetic analysis of a wide variety of transformations (acylation, $S_{N} A r$, silylation, solvolysis, Pd catalyzed C-S cross-coupling and cycloadditions) is demonstrated, highlighting both the versatility of the reactor and the benefits of performing kinetic analysis as a routine part of reaction optimization/development. Extension to the monitoring of multiple reactions simultaneously is also realized by operating the reactor with multiple reaction slugs at the same time.
\end{abstract}

\section{Introduction}

Measuring reaction kinetics is a powerful tool for enabling optimization, mechanistic investigation, and scale-up. ${ }^{[1]}$ Despite this, collection of kinetic data is often overlooked in lieu of less rigorous methods due to the laborious experimentation required. Recent advances in hardware, such as sampling tools and programmable liquid handling robotics, have sought to alleviate this problem. ${ }^{[2]}$ Mathematically simpler approaches to analyzing data, such as the visual variable time normalization method, have also been successful in reducing the barrier to studying kinetics in routine applications. ${ }^{[3]}$

Continuous flow systems offer numerous advantages over batch systems such as ease of automation, incorporation of online analytics, efficient mixing, and access to a larger range of temperatures and pressures. ${ }^{[4]}$ However, acquiring kinetic data is often considered an area where batch reactors are superior due to convenient sampling over time (Figure 1A). ${ }^{[5]}$ In contrast, time and space are coupled in a typical flow reactor, where a reaction's residence time is a function of the flow rate and distance traveled. Sampling over time must thus be carried out as a sequence of experiments with varied flow rate (Figure 1B). ${ }^{[6]}$ While effective, this approach is wasteful of both time and materials. To highlight this disadvantage, Blackmond and coworkers monitored the progress of an aldol reaction that required 40 minutes to reach completion. ${ }^{[5]}$ Due to the need to adjust flow rates and wait for

[a] Centre for Catalysis Research and Innovation, Department of Chemistry and Biomolecular Sciences, University of Ottawa, 10 Marie-Curie, Ottawa, Ontario, Canada, K1N 6N5

E-mail: stephen.newman@uottawa.ca

Supporting information for this article is given via a link at the end of the document. steady state before collection of each data point, a 5-fold increase in total reaction time and material consumption was required for flow compared to batch.

Solutions to this problem have so far focused on using stepped or gradient flow rates and model fitting software to circumvent the need to collect steady-state samples. ${ }^{[7]}$ However these have been limited by the complicated mathematics required, and application thus far restricted to relatively simple reactions. Furthermore, these methods sometimes suffer from an inability to unambiguously discriminate between potential reaction mechanisms without relying on chemical intuition.

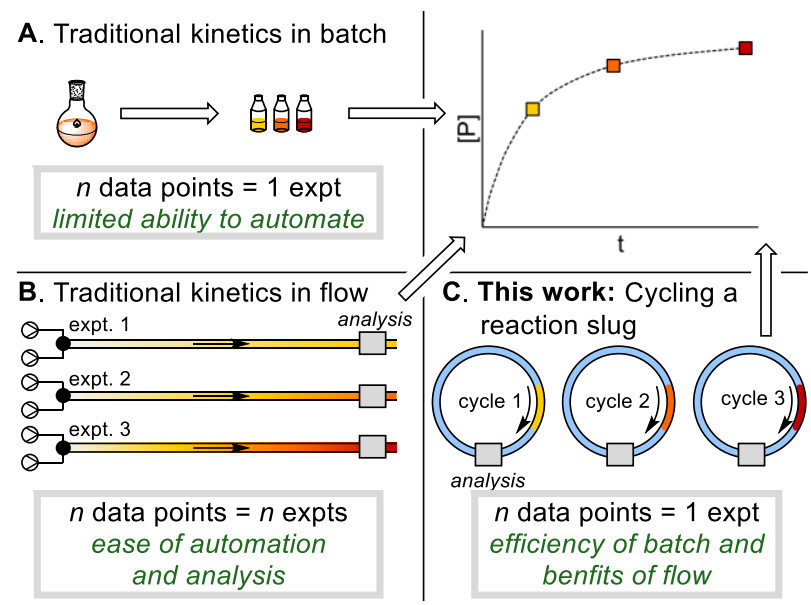

Figure 1. A) Generation of reaction profiles in batch is accomplished by aliquot sampling over time. B) Generation of reaction profiles in flow is accomplished by running a new steady-state experiment for each data point. C) This work: A flow reactor capable of analysing progress over time by cycling a reaction slug.

Given the growing number, diversity, and utility of advanced flow systems ${ }^{[8]}$ and the expanding scope of reactions that can be performed equally well or better when run in flow, ${ }^{[9]}$ we believed development of a simple and reliable method to obtain kinetic data from continuous systems would be invaluable. Flow reactors have proven particularly useful in the automated recovery and recycling of reaction components such as catalysts or auxiliaries through the implementation of recycling loops. ${ }^{[10]}$ With this inspiration, we envisioned cycling an entire reaction solution by performing the reaction in discrete slugs ${ }^{[11]}$ pushed by an inert carrier fluid (Figure $1 \mathrm{C})$. Analyzing the reaction once every loop provides reaction progress data.

Herein, we describe the design and implementation of such a reactor that enables straightforward acquisition of kinetic data. The versatility of this system is demonstrated through the study of a range of reaction types using varied solvents, temperatures, and kinetic analysis methods. The value of routinely performing kinetic analysis is additionally highlighted in the observation of nonintuitive rate behavior for seemingly simple transformations. The setup is assembled from commercially available components, and 
data quality was comparable or superior to batch sampling. We thus believe that flow kinetics via reaction cycling will be useful for both routine analysis and as a component in more complex automation platforms.

\section{Results and Discussion}

Sequential analysis of a cycling reaction slug is achieved by using two reactor coils linked by a 6-port, two-way valve and a selector valve with a minimum of 7-ports, six 6-positions. ${ }^{[12]}$ Simply changing the port connectivity and installing a modified rotor in the selector valve allows these valves to act as guides to control the fluid path. ${ }^{[13]}$ The principle of operation is that when the reaction slug is in coil $A$ it is directed to coil $B$ and when in coil $B$ it is directed back to coil A (Figure 2, see Figures $S 1$ in the supporting information for further details). Each time the slug is passed back and forth between the two reaction coils it travels through an intermediate zone where analysis is performed. While a range of online analysis tools can be envisioned, ${ }^{[14]}$ we elected to use a sampling valve that removes a small aliquot for off-line analysis, keeping the system cost and complexity low.

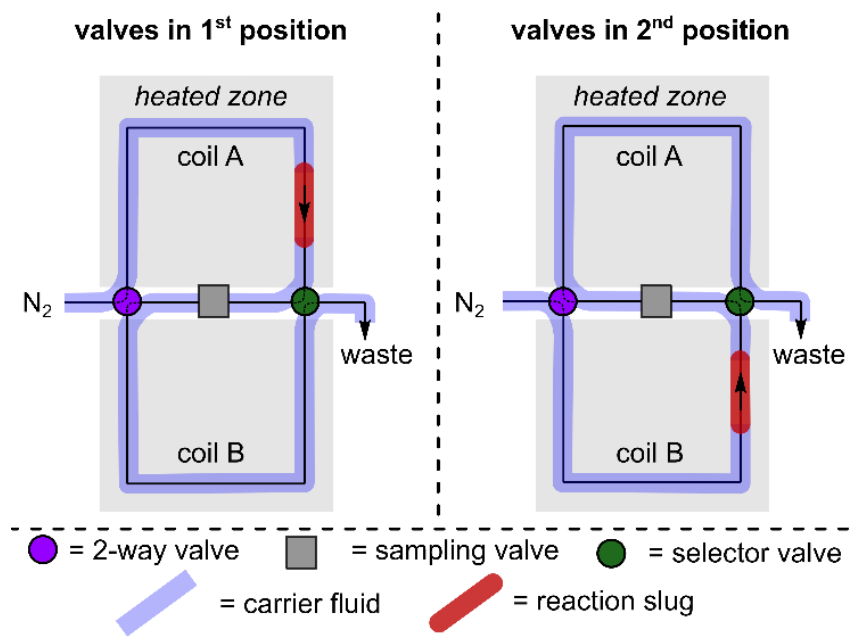

Figure 2. A schematic of the reactor coils and valves used to cycle a single reaction slug through a sampling valve multiple times, facilitating sequential sampling for reaction progress monitoring.

To validate the reactor, the room-temperature acylation reaction between benzoyl chloride (1) and benzyl alcohol (2) was studied. We selected $\mathrm{Bu}_{3} \mathrm{~N}(3)$ as the organic base to avoid solid handling issues, ${ }^{[9 e]} \mathrm{N}_{2}$ as the inert carrier fluid to move the reaction slug through the reactor, and the method of variable time normalization analysis (VTNA) ${ }^{[3]}$ to analyze the data (Figure 3 ). Experiments were performed both in a traditional batch set-up (i.e. a round-bottom flask) and with the cycling reactor. Identical results were obtained in both cases, finding first order behavior for both 1 (Figure 3A) and 2 (Figure 3B) and a partial reaction order of $\sim 0.5$ for to 3 (Figure $3 \mathrm{C}$ ). Related tertiary amine mediated acylation reactions are known to proceed by a nucleophilic catalyzed mechanism ${ }^{[15]}$, and the partial reaction order observed for 3 suggests that both a catalyzed and uncatalyzed reaction pathway may be operative. By normalizing to all reaction components, a straight line is obtained with a slope equal to the rate constant (Figure 3D). Replication of experiments in batch showed indistinguishable kinetic profiles (e.g. Figure 3E), confirming the validity and transferability of the collected data.

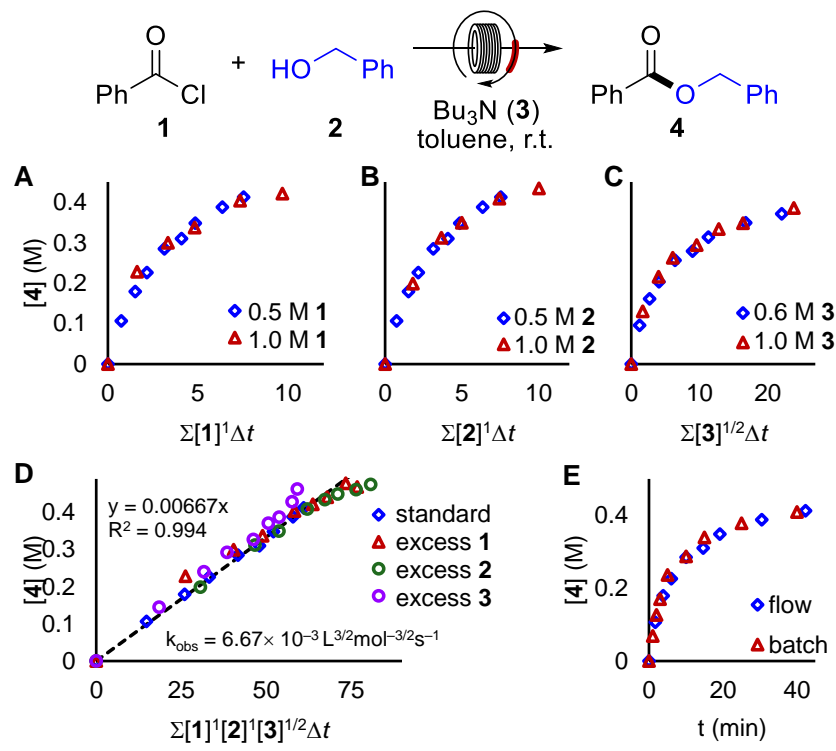

Figure 3. A) VTNA plot showing $1^{\text {st }}$ order in 1. B) VTNA plot showing $1^{\text {st }}$ order in 2. C) VTNA plot showing $\sim 0.5$ order in 3. D) VTNA plot to calculate rate constant. E) Overlay of data collected using flow reactor and batch data. Standard conditions: $0.5 \mathrm{M} \mathrm{1,0.5} \mathrm{M} \mathrm{2,} 0.6 \mathrm{M} 3$ in toluene, room temperature.

Satisfied that the reactor operated as desired, we next explored the ability to rapidly obtain kinetic data for a variety of reactions (Table 1). An $S_{N} A r$ reaction at $80{ }^{\circ} \mathrm{C}$ (entry 1 ) and a silylation at $0{ }^{\circ} \mathrm{C}$ (entry 2 ) were examined to assess the reactor performance over a range of temperatures. The flow reactor performed well in both cases, providing either equivalent or slightly superior data compared to parallel experiments in batch. The solvolysis of a secondary alkyl halide was probed using a pseudo-first order approach to distinguish between an $\mathrm{S}_{\mathrm{N}} 1$ or $\mathrm{S}_{\mathrm{N}} 2$ mechanism, as well as demonstrate the ability to perform an Eyring analysis by varying the reaction temperature (entry 3 ). A $\mathrm{Pd}$ catalyzed C-S cross-coupling reaction was examined using the method of initial rates to show the applicability towards airand moisture-sensitive chemistry and complex, multi-step reaction mechanisms (entry 4). Lastly, the ability to perform all necessary reactions simultaneously as consecutive slugs to maximize data collection efficiency was demonstrated with the analysis of a Diels-Alder cycloaddition (entry 5). The carrier fluid was also changed from $\mathrm{N}_{2}$ to $\mathrm{H}_{2} \mathrm{O}$ for this example to demonstrate the flexibility in choice of carrier solvent and the ability to conduct experiments above the atmospheric boiling point of the solvent $\left(\mathrm{CHCl}_{3}\right)$. 
Table 1. Reactions investigated using the flow reactor

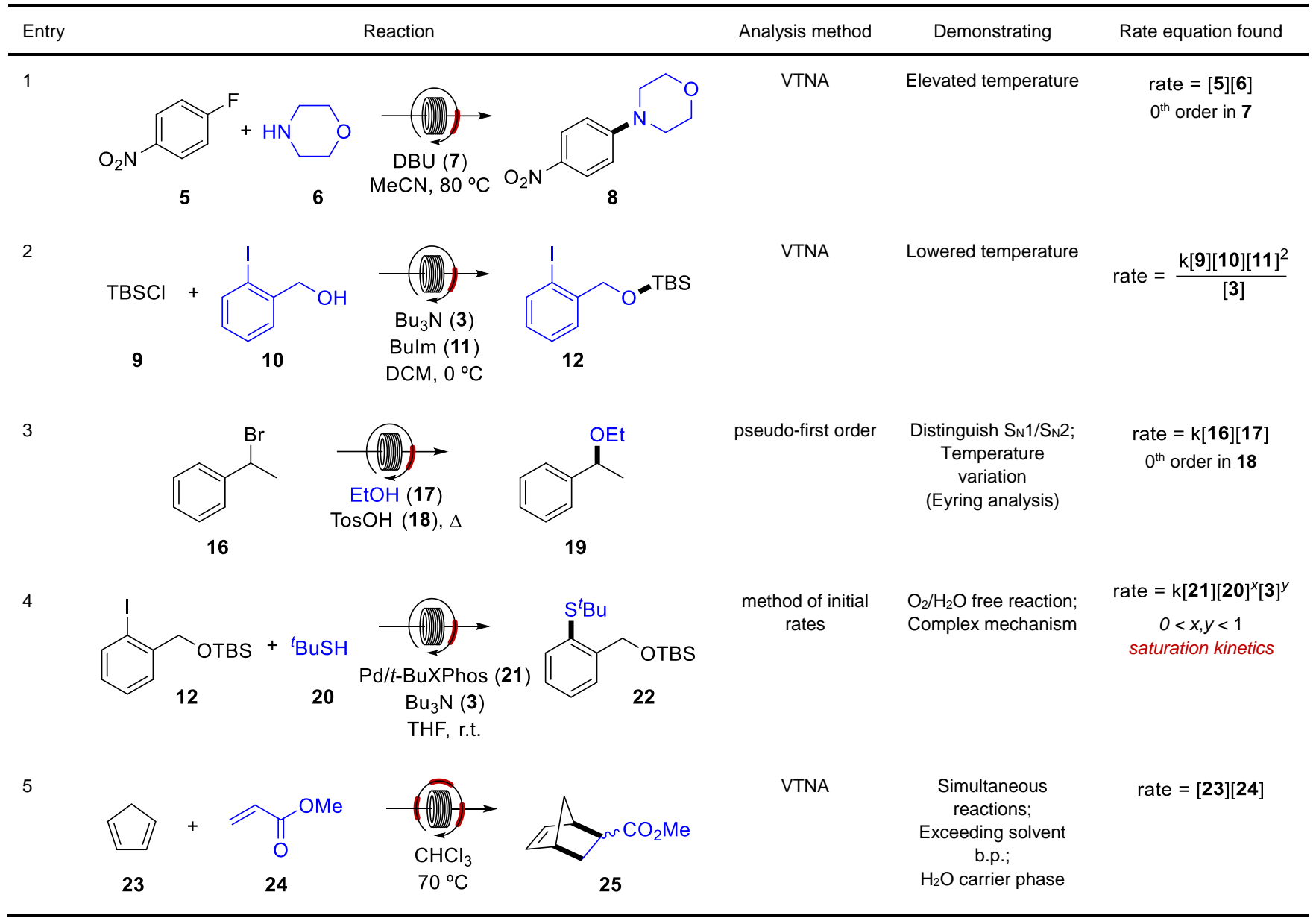

The observed rate equation for the $S_{N} A r$ reaction was as expected, with first order behavior observed for both electrophile 5 and nucleophile $\mathbf{6}$, and $0^{\text {th }}$ order for base 7 . The silylation of alcohol 10, however, showed non-intuitive second order behavior for the nucleophilic catalyst $\mathbf{1 1}$ and negative order with respect to base 3. A plausible reaction mechanism that accounts for the observed reaction orders is given in Scheme 1. There are two reaction steps leading to the product $\mathbf{1 2}$ that are comparably slow: attack of 1-butylimidazole (11) on TBSCI (9) to form a TBS-Bulm ${ }^{+}$ intermediate 13, and attack of 2-iodobenzyl alcohol (10) on 13 to lead to the product. In between these steps a non-productive but reversible equilibrium with the stoichiometric base (3) depletes the concentration of intermediate $\mathbf{1 3}$ by forming TBS-NBu${ }_{3}^{+}(\mathbf{1 4})$. The observed rate behavior contrasts with previous studies of TBS protection using DMAP/Et ${ }_{3} \mathrm{~N}$ as the catalyst/base. ${ }^{[15]}$ In this case, no inhibitory effect was observed, suggesting DMAP attack on TBSCI was the sole limiting step in that case.

For the solvolysis of alkyl bromide 16 an $\mathrm{S}_{\mathrm{N}} 2$ mechanism was found to be operative. First order behavior for $\mathbf{1 6}$ was found using integrated rate laws under pseudo-first order conditions (Figure 4A). First order behavior in $\mathrm{EtOH}$ (17) and zeroth order in acid 18 were determined by examining the effect of changing concentration on the observed rate constant (Table 2). Observing the effect of changing temperature on the rate constant allowed activation parameters to be calculated. An Eyring analysis of the data (Figure $4 \mathrm{~B}, \mathrm{k}=\mathrm{k}_{\mathrm{obs}} /[\mathrm{EtOH}]$ ) yielded values for the enthalpy $(18.9 \mathrm{kcal} / \mathrm{mol})$ and entropy $(-15.2 \mathrm{cal} /(\mathrm{mol} \cdot \mathrm{K}))$ of activation that were also consistent with an $\mathrm{S}_{\mathrm{N}} 2$ mechanism. ${ }^{[17]}$

Scheme 1. Postulated mechanism for the TBS protection of 10 mediated by 3 and 11.

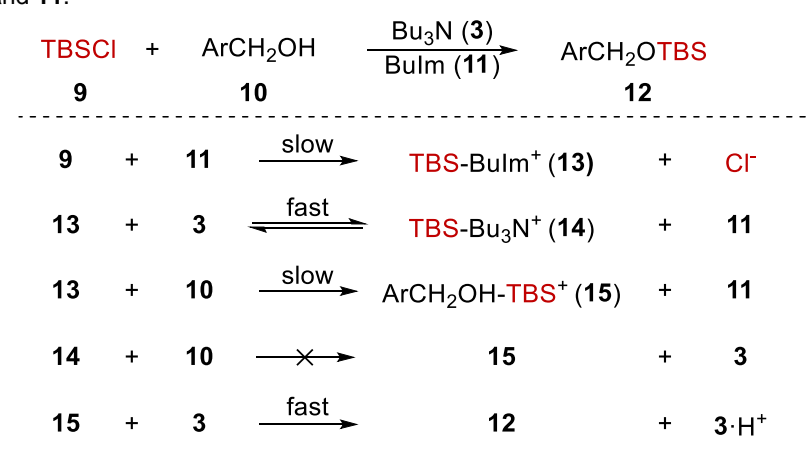


$\overbrace{16}^{\mathrm{Br}} \frac{\mathrm{EtOH}(17)}{\mathrm{TosOH}(\mathbf{1 8}), \Delta}$
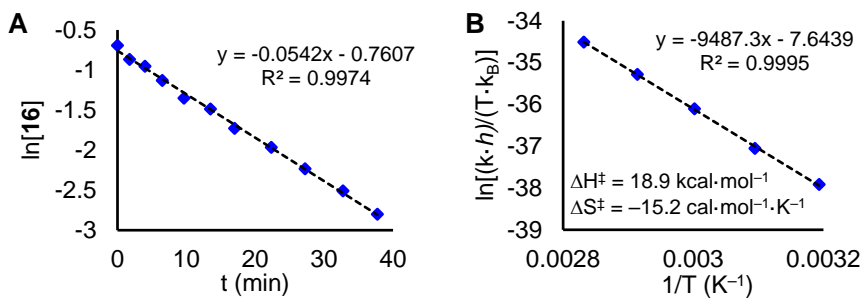

Figure 4. A) Linear integrated rate law plot showing first order in electrophile 16. B) Eyring plot. Standard conditions: $0.5 \mathrm{M} \mathrm{16}, 0.125 \mathrm{M} 18$ in $\mathrm{EtOH}, 70{ }^{\circ} \mathrm{C}$.

For the palladium catalyzed C-S cross-coupling recently reported by Buchwald and coworkers first order behavior was found for catalyst $\mathbf{2 1}$ (Figure 5A) and zeroth order for aryl halide 12 (Figure 5B), consistent with the previously identified LPd"ArX resting state. ${ }^{[18]}$ The reaction orders for thiol 20 and base 3 proved to be more complex, yielding curving log-log plots of initial rate vs. concentration (Figure $5 \mathrm{C}$ and $\mathrm{D}$ ). Both 20 and 3 exhibited saturation kinetics and Michaelis-Menten plots of the data fit well yielding $v_{\max }$ and $\mathrm{K}_{\mathrm{M}}$ values for each reagent (Figure $5 \mathrm{E}$ and $\mathrm{F}$ ). These data are consistent with a rate determining step of either deprotonation of the palladium bound thiol intermediate III or reductive elimination of the product from intermediate IV (see Figure 6). Subsequent DFT calculations concluded that reductive elimination is the rate limiting step (Figures S23-S25 in the supporting information).

Table 2. kobs values for the ethanolysis of 16 . $^{[a]}$

\begin{tabular}{llll}
\hline Entry & {$[\mathbf{1 8}](\mathrm{M})$} & {$[\mathbf{1 7}](\mathrm{M})$} & \multicolumn{1}{l}{$\mathrm{k}_{\mathrm{obs}}$} \\
\hline 1 & 0.125 & 16.0 & 0.0542 \\
2 & 0.0250 & 16.0 & 0.0599 \\
3 & 0.125 & $14.5^{[\mathrm{b}]}$ & 0.0484 \\
4 & 0.125 & $12.7^{[\mathrm{c}]}$ & 0.0384 \\
\hline
\end{tabular}

[a] All reactions $0.5 \mathrm{M}$ in 16. [b] 10:1 EtOH:t-BuOH as solvent. [c] 4:1 EtOH:t$\mathrm{BuOH}$ as solvent.
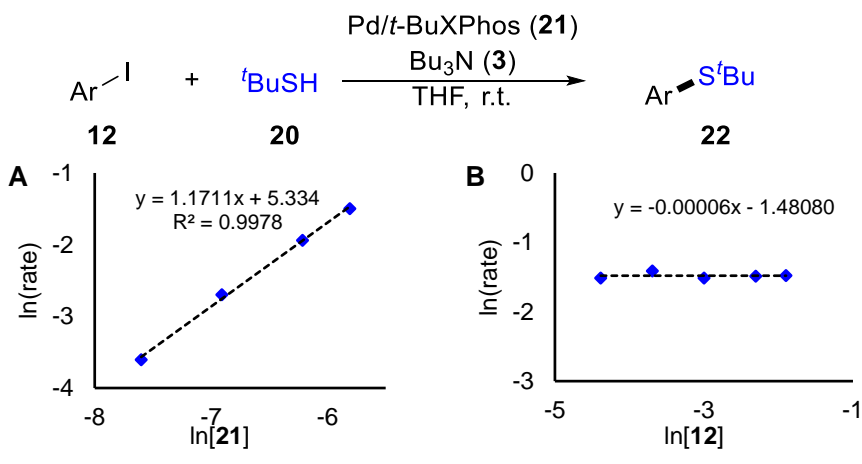
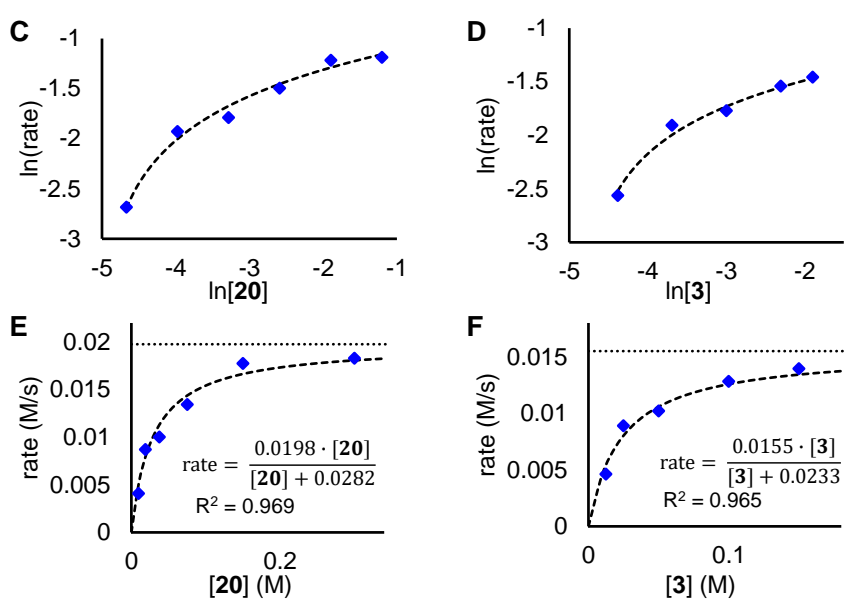

Figure 5. Initial rate kinetics for C-S cross coupling of Arl 12 and thiol 20 catalyzed by a $\mathrm{Pd} / t-\mathrm{BuXPhos}$ system. A) log-log plot of initial rate vs. [21], B) log-log plot of initial rate vs. [12], C) log-log plot of initial rate vs. [20], D) log-log plot of initial rate vs. [3], E) Michaelis-Menten plot of initial rate vs. [20], F) Michaelis-Menten plot of initial rate vs. [3]. Standard conditions: $50 \mathrm{mM} \mathrm{12,} 75$ $\mathrm{mM} 20,100 \mathrm{mM} \mathrm{3,3} \mathrm{mM} 21$ in THF, room temperature.

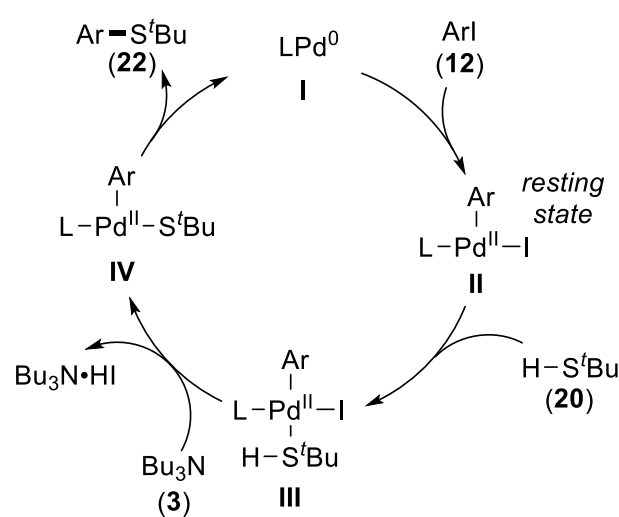

Figure 6. Putative catalytic cycle for the Pd catalyzed C-S bond formation. $\mathrm{L}=$ $t$-BuXPhos, Arl = 12

While experiments discussed thus far featured single reaction slugs cycled and analyzed over time, the ability to perform multiple reactions simultaneously, and therefore generate all data necessary for kinetic analysis at the same time, was envisioned. This is especially appealing for slow reactions, where the time required to collect all data is particularly tedious. The Diels-Alder reaction between cyclopentadiene (23) and methyl acrylate $(24)$ required $\sim 3 \mathrm{~h}$ to reach $>80 \%$ conversion at $70{ }^{\circ} \mathrm{C}$, making it an ideal candidate to demonstrate this ability. The volume of the residence coils was increased and the three necessary reactions (i.e., "standard conditions", and excess in each reagent) were injected as sequential slugs in a way that allowed the flow path to be altered each time all slugs were in the same residence coil (Figure 7). The carrier phase was also changed from $\mathrm{N}_{2}$ to water to allow the reaction to be conducted above the boiling point of the solvent through application of backpressure. In this way, all necessary data for kinetic analysis 
was collected in $\sim 3 \mathrm{~h}$, as opposed to the $\sim 9 \mathrm{~h}$ that would have been required if running each reaction consecutively with this flow reactor, or the $\sim 60 \mathrm{~h}$ that would be required to collect equivalent data using the traditional steady-state approach of changing the flow rate to change residence time for each data point.

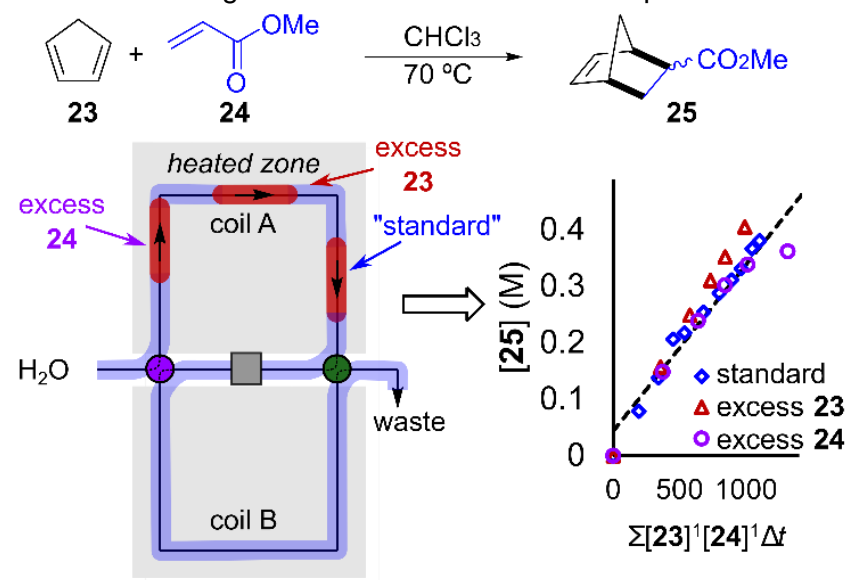

Figure 7. Operating with multiple sequential reaction slugs allows monitoring of multiple reactions simultaneously.

While the system has some limitations, in e.g., handling multi-phasic or extremely fast reactions, the ability to efficiently collect reaction progress data in flow, and applicability to a wide range of reactions and conditions holds promise for wide applicability.

\section{Conclusion}

We have developed a reactor that allows reaction progress to be monitored over time from a continuously cycling reaction slug. The reactor performance was assessed over a wide range of temperatures $\left(0-80^{\circ} \mathrm{C}\right.$ ), solvents (toluene, $\mathrm{MeCN}, \mathrm{DCM}$, EtOH, $\mathrm{THF}, \mathrm{CHCl}_{3}$ ) and reactions (acylation, $\mathrm{S}_{\mathrm{N}} \mathrm{Ar}$, silylation, ethanolysis, C-S cross-coupling, Diels-Alder). The ability to use the reactor to distinguish between potential reaction mechanisms and determine activation parameters was demonstrated. Lastly, the ability to perform multiple reactions simultaneously as consecutive reaction slugs was shown with the kinetic analysis of a Diels-Alder reaction.

The application of the reactor to collect data for a variety of different methods of kinetic analysis was also demonstrated, including variable time normalization analysis, pseudo-first order kinetics, Eyring plots and the method of initial rates. We believe the development of this reactor marks the first true equivalent in flow to the generation of reaction progress data in batch, where analysis over time from a single reaction solution is the most efficient strategy with regards to both time and material consumption. Therefore, since this reactor combines both the efficiency of the traditional batch sampling strategy with the benefits of flow, we believe this platform will lower the impediment to routine kinetic analysis, through both stand-alone operation and in combination with reaction platforms to automate kinetic experiments and data generation.

\section{Acknowledgements}

Financial support for this work was provided by the University of Ottawa, the National Science and Engineering Research Council of Canada (NSERC), and the Canada Research Chair program. The Canadian Foundation for Innovation (CFI) and the Ontario Ministry of Economic Development and Innovation are thanked for essential infrastructure. Debasis Mallik (CCRI flow chemsitry facility) and Peter Zhang (Vici Valco) are thanked for programming the drivers used to control the sampling valves. $R$. J. S. thanks NSERC for a CGS-D award.

\section{Conflicts of Interest}

The authors declare no conflict of interest.

Keywords: kinetics $\cdot$ flow $\cdot$ variable time normalization analysis - initial rates $\cdot$ pseudo-first order $\cdot$ acylation $\bullet \mathrm{S}_{N} \mathrm{Ar} \cdot$ silylation $\bullet$ Diels-Alder $\cdot \mathrm{C}$-S bond formation

[1] a) J. W. Moore, R. G. Pearson, Kinetics and Mechanism, 3 ed., Wiley New York, 1981; b) R. I. Masel, Chemical Kinetics and Catalysis, Wiley, New York, 2001; c) D. G. Blackmond, Angew. Chem. Int. Ed. 2005, 44, 4302-4320; d) D. G. Blackmond, J. Am. Chem. Soc. 2015, 137, $10852-10866$.

[2] a) A. M. Fermier, A. R. Oyler, B. L. Armstrong, B. A. Weber, R. L. Rodriguez, J. V. Weber, J. A. Nalasco, J. Assoc. Lab. Automation 2002, 7, 83-88; b) R. Chung, D. Yu, V. T. Thai, A. F. Jones, G. K. Veits, J Read de Alaniz, J. E. Hein, ACS Catal. 2015, 5, 4579-4585; c) C Rougeot, H. Situ, B. H. Cao, V. Vlachos, J. E. Hein, React. Chem. Eng 2017, 2, 226-231; d) T. C. Malig, J. D. B. Koenig, H. Situ, N. K. Chehal P. G. Hultin, J. E. Hein, React. Chem. Eng. 2017, 2, 309-314; e) A. M. Fermier, A. R. Oyler, B. L. Armstrong, J. V. Weber, J. Nalasco (OrthoMcNeil Pharmaceutical, Inc., USA .), WO patent 2002077614 A2, 2002

[3] a) J. Burés, Angew. Chem. Int. Ed. 2016, 55, 2028-2031; b) J. Burés, Angew. Chem. Int. Ed. 2016, 55, 16084-16087.

[4] a) J. P. McMullen, K. F. Jensen, Annu. Rev. Anal. Chem. 2010, 3, 19-42 b) R. L. Hartman, J. P. McMullen, K. F. Jensen, Angew. Chem. Int. Ed. 2011, 50, 7502-7519; c) S. G. Newman, K. F. Jensen, Green Chem 2013, 15, 1456-1472; d) S. V. Ley, D. E. Fitzpatrick, R. J. Ingham, R. M. Myers, Angew. Chem. Int. Ed. 2015, 54, 3449-3464.

[5] F. E. Valera, M. Quaranta, A. Moran, J. Blacker, A. Armstrong, J. T. Cabral, D. G. Blackmond, Angew. Chem. Int. Ed. 2010, 49, 2478-2485.

[6] For example: a) J. P. McMullen, K. F. Jensen, Org. Process Res. Dev. 2011, 15, 398-407; b) A. Gholamipour-Shirazi, C. Rolando, Org. Process Res. Dev. 2012, 16, 811-818; c) D. M. Roberge, C. Noti, E. Irle, M Eyholzer, B. Rittiner, G. Penn, G. Sedelmeier, B. Schenkel, J. Flow Chem. 2014, 4, 26-34; d) C. A. Hone, A. Boyd, A. O'Kearney-McMullan, R. A. Bourne, F. L. Muller, React. Chem. Eng. 2019, 4, 1565-1570.

[7] a) S. Mozharov, A. Nordon, D. Littlejohn, C. Wiles, P. Watts, P. Dallin, J. M. Girkin, J. Am. Chem. Soc. 2011, 133, 3601-3608; b) J. S. Moore, K. F. Jensen, Angew. Chem. Int. Ed. 2014, 53, 470-473; c) S. Schwolow, F. Braun, M. Rädle, N. Kockmann, T. Röder, Org. Process Res. Dev. 2015, 19, 1286-1292; d) C. A. Hone, N. Holmes, G. R. Akien, R. A Bourne, F. L. Muller, React. Chem. Eng. 2017, 2, 103-108; e) K. C. Aroh, K. F. Jensen, React. Chem. Eng. 2018, 3, 94-101.

[8] a) B. J. Reizman, K. F. Jensen, Acc. Chem. Res. 2016, 49, 1786-1796; b) S. Mascia, P. L. Heider, H. Zhang, R. Lakerveld, B. Benyahia, P. I. Barton, R. D. Braatz, C. L. Cooney, J. M. B. Evans, T. F. Jamison, K. F. Jensen, A. S. Myerson, B. L. Trout, Angew. Chem. Int. Ed. 2013, 52, 12359-12363; c) A. Adamo, R. L. Beingessner, M. Behnam, J. Chen, T. 
F. Jamison, K. F. Jensen, J.-C. M. Monbaliu, A. S. Myerson, E. M. Revalor, D. R. Snead, T. Stelzer, N. Weeranoppanant, S. Y. Wong, P. Zhang, Science 2016, 352, 61-67; d) D. Perera, J. W. Tucker, S. Brahmbhatt, C. J. Helal, A. Chong, W. Farrell, P. Richardson, N. W. Sach, Science 2018, 359, 429-434; e) C. W. Coley, D. A. Thomas, J. A. M. Lummiss, J. N. Jaworski, C. P. Breen, V. Schultz, T. Hart, J. S. Fishman, L. Rogers, H. Gao, R. W. Hicklin, P. P. Plehiers, J. Byington, J. S. Piotti, W. H. Green, A. J. Hart, T. F. Jamison, K. F. Jensen, Science 2019, 365 eaax1566.

[9] a) M. B. Plutschack, B. Pieber, K. Gilmore, P. H. Seeberger, Chem. Rev. 2017, 117, 11796-11893; b) J. C. Pastre, D. L. Browne, S. V. Ley Chem. Soc. Rev. 2013, 42, 8849-8869; c) S. Newton, C. F. Carter, C. M. Pearson, L. D. Alves, H. Lange, P. Thansandote, S. V. Ley, Angew. Chem. Int. Ed. 2014, 53, 4915-4920; d) K. P. Cole, J. M. Groh, M. D. Johnson, C. L. Burcham, B. M. Campbell, W. D. Diseroad, M. R. Heller, J. R. Howell, N. J. Kallman, T. M. Koenig, S. A. May, R. D. Miller, D. Mitchell, D. P. Myers, S. S. Myers, J. L. Phillips, C. S. Polster, T. D. White, J. Cashman, D. Hurley, R. Moylan, P. Sheehan, R. D. Spencer, K. Desmond, P. Desmond, O. Gowran, Science 2017, 356, 1144-1150; e) S. K. Kashani, R. J. Sullivan, M. Andersen, S. G. Newman, Green Chem. 2018, 20, 1748-1753; f) M. G. Russell, T. F. Jamison, Angew. Chem. Int. Ed. 2019, 58, 7678-7681.

[10] a) I. Vural Gursel, T. Noel, Q. Wang, V. Hessel, Green Chem. 2015, 17, 2012-2026; b) R. J. Sullivan, S. G. Newman, Chem. Sci. 2018, 9, 2130-2134.

[11] For examples of reactions performed in discrete slugs or droplets see: a) H. Song, D. L. Chen, R. F. Ismagilov, Angew. Chem. Int. Ed. 2006, 45, 7336-7356; b) M. Sesen, T. Alan, A. Neild, Lab Chip 2017, 17, 2372-2394; c) T. S. Kaminski, P. Garstecki, Chem. Soc. Rev. 2017, 46, 6210-6226; d) E. S. Isbrandt, R. J. Sullivan, S. G. Newman, Angew. Chem. Int. Ed. 2019, 58, 7180-7191; e) N. Candoni, R. Grossier, M. Lagaize, S. Veesler, Annu. Rev. Chem. Biomol. 2019, 10, 59-83; f) B. J. Reizman, K. F. Jensen, Chem. Commun. 2015, 51, 13290-13293; g) Y.J. Hwang, C. W. Coley, M. Abolhasani, A. L. Marzinzik, G. Koch, C. Spanka, H. Lehmann, K. F. Jensen, Chem. Commun. 2017, 53, 6649-6652.

[12] Valves with a larger number of ports could also be used, providing the same fluid connectivity is achieved through selection/fabrication of appropriate routers. For example, in our case we had access to an 11port, 10-position selector valve which was used instead of a 7-port, 6position selector valve by fabrication of a suitable router. For full details see the SI.

[13] A simple, minor modification, 100 USD for a modified router.

[14] G. A. Price, D. Mallik, M. G. Organ, J. Flow Chem. 2017, 7, 82-86.

[15] a) P. Hubbard, W. J. Brittain, J. Org. Chem. 1998, 63, 677-683; b) A. J. Oosthoek-de Vries, P. J. Nieuwland, J. Bart, K. Koch, J. W. G. Janssen, P. J. M. van Bentum, F. P. J. T. Rutjes, H. J. G. E. Gardeniers, A. P. M. Kentgens, J. Am. Chem. Soc. 2019, 141, 5369-5380.

[16] P. Patschinski, C. Zhang, H. Zipse, J. Org. Chem. 2014, 79, 8348-8357.

[17] For a discussion of typical values for activation enthalpies and entropies see: E. V. Anslyn, D. A. Dougherty, Modern Physical Organic Chemistry, University Science, Sausalito, 2006

[18] J. Xu, R. Y. Liu, C. S. Yeung, S. L. Buchwald, ACS Catal. 2019, 9, 6461-6466. 


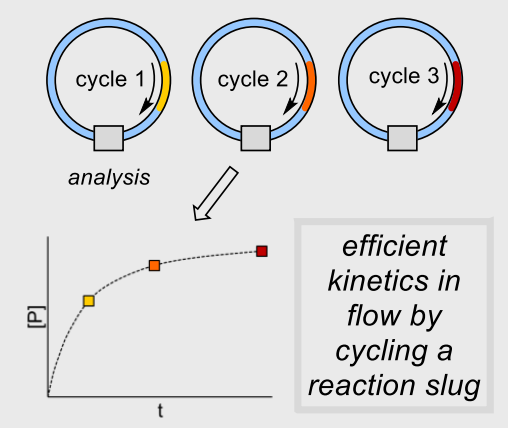

